\title{
DEUX TITRES BOUDDHIQUES PORTÉS PAR DES RELIGIEUX NESTORIENS
}

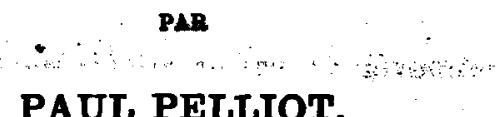

PAUL PELLIOT.

Le P. Havret est mort en 1901 sans aroir pu mettre la dernière main au travail si intéressant et si minutieux qu'il avait consacré à l'inscription chrétienne de Si-ngan-fou. Toutefois, dès 1897, ') il avait obtenu du $P$. Cheikho un déchiffrement et une traduction des notes syriaques qui se trouvent sur les diverses faces de la stèle, et les six pages de cette étude ont été jointes au fascicule où les jésuites de $Z i-k a-w e i$ ont pieusement édité les notes laissées par leur regretté confrère. ${ }^{2}$ )

Le premier personnage dont ait en à s'occuper le P. Cheikho est l'auteur même de l'inscription, le religieux Adam, qui avait pris le nom chinois de 景掙 King-tsing. ${ }^{3}$ ) En syriaque, cet auteur

1) Cf. Variétés sinologiques, $\mathrm{n}^{\circ}$. 12, Havret, La stèle chrétienne de Si-ngan-fow, II partie, 1897, p. 210.

2) Variétés sinologiques, $\mathrm{n}^{\circ}$. 20, Havret, La stèle clirétienne de Si-ngan-fou, III* partie, 1902. Wue nouvelle étude sur la stèle de. Si-ngan-fon, publiée par un Japonais, est signaléc dans B.E.F.E.-O, X, 731; il est tout à fait improbable qu'elle dise rien de la partie syriaque.

3) Je rappe!le que King-tsing est encore connu, grâce à une heureuse trouvaille de M. 'Takakusu, comme traducteur d'un traité bouddhique sur les sir päramitā (cf. T'oung Puo, VII, 589-591). Son nom se rencontre en outre dans la note finsle de l'Eloge de la Sainte T'rinité que j'ai retrouvé dans la grotte de Touen-houang et qui est aujourd'hui 
est qualifié de «Adam, prêtre et chorévêque et papas de Chine»; telle est du moins la version du $P$. Cheikho.

Un seul titre fait difficulté, celui de papas. Le P. Cheikho expliquait au P. Havret que, adans les premiers siècles de l'Eglise, le nom de pape était donné indifféremment aux patriarches, aux érêques et même aux prêtres s; et il rappelait les papas des Grecs modernes et les popes russes. ") Le P. Havret s'est rangé à l'avis du P. Cheikho. Cette solution, en dehors de toute autre considération, ${ }^{2}$ ) prête cependant à une objection capitale: l'inscription de Si-ngan-fou ne donne pas papas, mais incontestablement papši, ${ }^{3}$ ) qui ne se ramène pas à papas. C'est ce qui arait conduit le $P$. Heller ${ }^{4}$ ) et $M^{g r}$ Lamy ${ }^{5}$ ) à supposer pour papši, qui n'est pas syriaque, une origine étrangère, en fait chivoise.

conservé à la Bibliothèque Nationale (cf $B, E . F . E .-O .$, VIII, 519; le texte a été édité en 1909-

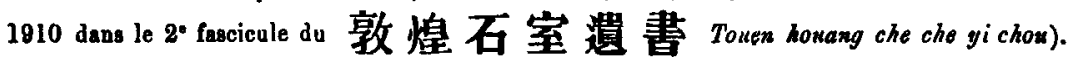

1) Stele chrétionne, III partie, p. 8.

2) Ce titre de «papas * appliqué a Adam, prêtre et chorévêque, c'est-à-dire n'ayant même pas le caractère épiscopal, ne va pas de soi. En tout cas, il faut absolument renoneer à rapprocher le titre donné ici à Adam de celui de fa-zoang, "roi de la loi ", ou plutôt

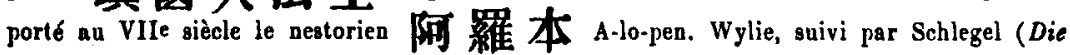
chinesische Inschrift auf dem uigurischen Denkmal in Karabalgassun, p. 64), s'est mépris ì ce sujet.

3) On fapłi, l'Geriture syriaque de l'inscription ne distinguant pas entre $f$ et $p$.

4) Heller, Das nestorianische Denknal in Singan-fu (dans Zeitschr. für Katb. Theol., Innsbrück, 1885, pp. 111, 123); Proleqomena zu einer newen Ausgabe der nestorianischen Inschrift won Singax-fw, dans Verhandl. des VII intern. Orient.-Congresses gehalten in Wien im Jahre 1886, Vienne, Hölder, 1889, pp. 45-46; Beleuchtung der Bemerkungen Kühnert'd zu meinen Schriften über das nestorianische Denkmal zu Singan-fu, dans $W . Z$. K. M., 1895, 318-313; Das nestorianische Denkmal in Singan-fu, Budapest, 1897, in-4 $4^{\circ}$, pp. 42-43. Il me paraît inutile de discuter ici les objections confuses et contradictoires que M. Kühnert opposées an P. Heller dans W.Z.K.M., 1895, pp. 2E-43.

5) Mgr. Iamy, Le monumext ehrétion de Si-ngan fou, en collaboration avec A. Gueluy (forme le 6" fasc. du t. LIII des Mém. de l'Acad. roy. de Belgique), 1897, p. 99. Mgr. Lamy hésitait, d'après la pholographie dont il se serrait, entre apapaschis et apapaschahs. Le P. Havret (Stèle chrélienne, III, 8) lai répondit qu'il aurait pu consulter la photolithographie des l'ariétés sinologiques, wdont leg traits sont parfaitement formésn. Ceci est exact, 
Mgr Lamy ne fit pas d'hypothèse personnelle. Par contre, le P. Heller s'adressa à von der Gabelentz, qui lai suggéra 法師 fa-che $\left({ }^{*} f a ̆ p-s^{\prime} i\right)$ ou 法士 fa-che $\left.\left({ }^{*} f a{ }^{\prime} p-j^{\prime} i\right) .{ }^{1}\right)$ Le P. Heller garda fa $\left({ }^{*} f a p\right)$, mais rejeta 師 che et 士 che, parce que ces expressions signifieraient «maitre», et que, parmi les 76 religieux nommés an bas et dans les marges de l'inscription, et dont beaucoup devaient être des «maîtres», il était inadmissible que ce titre de fa-che, -maîtres, fút attribué au seul Adam. Après avoir songé à une combinaison 法史 $f a-c h e$, qui surait signifié cannaliste de la Loin, le P. Heller se décida en faveur de 法司 fa-sseu, disant que sseu se prononce également ši, et interprétant cette expression par asurveillant de la Loi».

Le P. Havret n'accepta pas l'hypothèse du P. Heller, pour deux raisons: d'abord à cause de ce que le P. Cheikho lui disait de papas, et aussi parce que cà l'époque des T'ang, nous ne rencontrons pas une seule fois un caractère chinois prononcé aujourd'hui $f a$, pris pour le son pa ou pap dans les transcriptions bouddhiques faites à Si-ngan-fous. En note, le P. Havret ajoutait, en s'appuyant sur la Méthode pour déchiffrer et transorire les noms sanscrits de Stanislas Julien, que cles caractères qui alors représentaient le pa sanscrit, étaient 波, 鎬, 践, 般, 巴 et autres semblables》.

mais ces traits donnent papłi, et on ne comprend pas pourquoi le P. Cheikho, qui écrit correctement papši en syriaque et en caractères bébreux, transcrit papas en caractères latins.

1) Heller, Das nestorianische Denkmal, 1897, p. 42; je ne cite que ce dernier travail, où le $\mathrm{P}$. Heller a développé ses hypothèses; en réalité elles se trouvent déjà dans son étude de 1885. Von der Gabelentz n'avait indiqué la torme ancienne que de 漠 *fáp; pour les deux sutres caractères, il les edt las uniformément $\mathfrak{s}_{i}$, ayant toujours négligé la différence essentielle des initiales sourdes et sonores, qui se complique ici, dans un des denx cas, d'un passage d'explosive à spirante. La marque de brève employée par von der Gabolentz dans * făp est certainement inutile, et pent-être fautive. Le P. Heller a de son côté restitué des "prononciations anciennes" poar les noms chinois de tous les moines, et laimême reconnait (p. 59) que ces restitutions sont parfois hypothétiques; elles sont on réalité de la plus déconcertante fantaisie. Je note par l'apostrophe le yod; cette notation est provisoire. 
On a vu plus hant sar quelle confusion de formes reposait l'opinion du P. Cheikho. L'argument tiré de la Méthode de Julien ne raut pas mieux. Cette Méthode, qui a rendu des services, est aujourd'hui tout à fait surannée; elle coupe arbitrairement les portions de mots étrangers que chaque caractère chinois est censé transcrire, et ne tient aucun compte de la prononciation qu'avaient ces caractères à l'époque des T'ang. Des signes comme 波 po

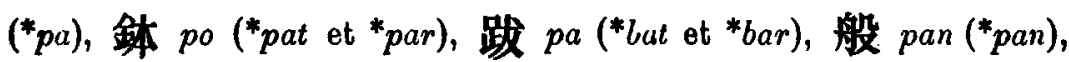
巴 $p a\left({ }^{*} p a\right)$ transcrivent en réalité des mots ou portions de mots très différents, qui ne sont pa que pour le premier et le dernier. Et puis, il ne s'agissait pas de rendre en chinois un son étranger pa ou pap, mais éventuellement de transcrire en syriaque un son $f a$, ou plutôt * fap. Surtout le P. Havret ne paraît pas avoir remarqué que l'écriture syriaque de l'inscription ne distiugue pas entre $f$ et $p$, et que c'est le même signe que le P. Cheikho transcrit tantôt par $p$, tantôt par $f ;$ papši peut donc bien être fapši, et l'est certainement si ce mot transcrit une expression chinoise commençant par 法 $f a\left({ }^{*} f a p\right)$.

Or je crois bien que tel est le cas, mais autrement que ne l'entend le P. Heller. Son 法司 fa-sseu, esurveillant de la Lois, est une expression qu'il forge de toutes pièces; elle ne s'est, je crois, jamais rencontrée, an moins dans cet emploi. De plus, il est inexact que 司 sseu ait aussi une prononciation $̌ s i$ la prononciation ancienne da caractère est $*_{s y}$, en notant par $y$ une voyelle de timbre sourd qui ne yodisait pas la sifflante précédente comme dans les mots de type si $\left({ }^{*}{ }^{\prime} i\right)$; jamais, dans les temps historiques, ce mot n'a été prononcé arec la chuintante de pap̧̌i (fapši). La seconde objection ne vaut pas contre l'hypothèse à laquelle le $\mathrm{P}$. Heller avait tout

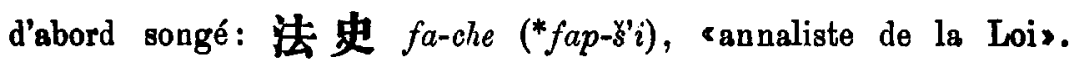
Ceci serait phonétiquement possible, mais l'autre difficulté reparaît: 
l' anualiste de la Lois est lui aussi une combiuaison arbitraire, et encore moins admissible que le surveillant de la Lois.

Restent les deux hypothèses de vou der Gabelentz. One forme 法士 $f a$-che $\left({ }^{*} f a p-j{ }^{\prime} i\right)$ n'est pas inpossible on chinois, et l'objection phonétique ne serait pas insurmontable. Mais ce n'est pas là une combinaison usuelle, et nous avons mieux avec 法師 fa-che $\left({ }^{*}\right.$ fap-š $\left.i\right)$. Cette fois, tout concorde. La correspondance phonétique est parfaite, et nous tenons enfin, ce qui est presque l'essentiel, une forme réellement et constamment usitée dans la langue religiense de la Chine. saître de la Lois, c'est le titre des maîtres bouddhistes. Que des nestoriens l'aient adopté, nous n'en serons pas surpris quand nous leur voyons prendre, dans la partie chinoise de l'inscription, les titres non moins bouddhiques de 僧 seng, smoines, qui est le sanscrit sangha, ou de 大德 ta-tö, sgrande vertus, qui répond régulièrement au sanscrit bhadanta. Et si le P. Heller objecte qu'il derrait y avoir, dans la longue liste des religieux nestoriens, d'autres cmaitres de la Lois qu' Adam, j'avoue qu'en tout état de cause cet argument négatif ne me paraît pas avoir grande valeur. Mais nous devous aussi nous. rappeler le rôle que le prêtre nestorien Adam a joué comme traducteur d'œurres bouddhiques, et nous pourrous admettre que c'est à ces relations avec les cmaîtres de la Loi, bouddhistes qu'il devait d'avoir pris on reçu pour lui-même leur titre le plus ordinaire ${ }^{1}$ ).

Ainsi papši (fapši) ne serait antre que le titre bouddhiste de 法師 fa-che $\left({ }^{*} f a p-\varsigma^{\prime} i\right)$, smaitre de la Lois. La vraisemblance de cet emprunt me paraît corroborée par un second terme employé

1) Ce titre de amaître de la Loi. est appliqué à un religieux manichéen dans l'inseription de Kara-balgasoun (cf. Sehlegel, Die chinesische Inschrift auf dem wigurischen Denkmal in Karabalgassun, Helingfors, 1896, p. 18). In ce qui concerne Adam, je me demande si précisément le génitif *de Chine. qui suit le titre de fapłi ne porte pas exclusirement sur ce dernier titre, et n'indique pas par là-mêne que c'est en Chine qu' Adam, prêtre et chorévêque, arait reçu cette nourelle appellation. 
dans la partie syriaque de notre inscription. Sur la face de droite, nous trouvons indiqué, dans la traduction du P. Cheikho, un "Marsargis, prêtre et chorévêque de Schiangatsoa»; ce «Schiangatsoa» n'est pas autrement expliqué. Le P. Heller a lu «schiangtsue», et a supposé un original chinois: ‘Ce mot, dit-il, ne peut pas être un nom de lieu, comme «chorévêque de Schiangtsue», ni un second titre; dans le premier cas, il faudrait avoir la marque du génitif, et dans l'autre le mot "et». Le mot chinois est peut-être 郎主 hiang-ču, ou 楖 首 hiang-sau (Canton), hiang-so, hiang-tschü (Swatow),

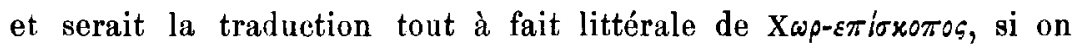
pouvait constater que hiang, "village», s'est prononcé jadis šiang.»

L'objection faite par le P. Heller contre «Schiangtsue», nom de lieu, me paraît tout à fait sérieuse; la marque du génitif, en fait $d$ placé devant le mot au génitif, est nécessaire, et n'est jamais omise dans l'inscription. Il y a moins de difficulté à admettre un second titre, qui, étant au même cas que le précédent, peut, à la rigueur, se passer de set». Même l'hypothèse du P. Heller revient un peu à cela; son hiang-ču, mis comme une sorte de traduction à la suite de chorévêque, sera difficilement accepté comme une simple apposition. Bien plus, on trouve, sur la face principale de la stèle, la mention de Mar Hnanišo, "catholicos et patriarche»; le P. Cheikho tout comme le P. Heller ${ }^{1}$ ) admettent bien qu'il s'agit de deux titres; or ils ne sont pas séparés par la conjonction «et».

Je pense donc que la forme syriaque dissimule un titre, et que ce titre non syriaque est chinois. Mais je ne crois pas au schef de village» du P. Meller. On attend un titre religieux. De plus hiang-tchou et hiang-cheou sont l'aboutissement moderne, à peine altéré, de prononciations anciennes $h^{\prime} a \dot{n}-\check{c}^{\prime} u$ et $h^{\prime} a \dot{n}-s^{\prime} e u^{2}$ ), auxquel-

1) Cheikho, loc. laud., pp. 1, 6; Heller, loc. laud., p. 36.

2) Le yod, qui affaiblissait $a$ en $\ddot{a}$ devant $m$ et $n$, n'a jamais produit ce résultat devant $\dot{n}$.

Deel XII 
les ne saurait répondre «schiangtsue». Ici encore, il me semble qu'on doit chercher du côté du bouddhisme. Le syriaque porte en réalité šiangtsua: or c'est exactement ainsi qu'on peut s'attendre à voir transerit, par quiconque avait l'oreille un peu fine, le titre de 上 座 chang-tso, qui répond au sanserit sthavīra et désigne le supérieur des couvents. La prononciation ancienne de chang-tso est ž $a \dot{n}$ tswa, car les tables du K'ang hi tseu tien attestent, d'une part que toutes les chuintantes étaient yodisées, d'autre part que 座 tso rentre dans la catégorie des mots prononcés "la bouche fermée. (合口), c'est-à-dire avec insertion d'une semi-voyelle labiale. La correspondance est si parfaite que la solution me paraît s'imposer.

- Le chorévêque Marsargis devait être le supérieur d'un couvent nestorien. ${ }^{1}$ )

Si mes hypothèses sont justes, il en résulterait qu'à côté des titres bouddhistes que nous voyons employés en chinois par les nestoriens, ceux-ci avaient encore adopté, même dans leur langue maternelle, quelques titres usuels du pays où ils s'étaient établis, et en particulier ceux de fa-che, «maître de la Loi», et de changtso, supérieur de couvent».

1) Le chang-tso ne se confond pas avec le 考 主 sseu-tchou, "maître du temple". Il y a aussi un sseu-tchou mentionné dans l'nscription de Si-ngan-fou, et qui porte le titre de $大$ 学 卿 t'ai-tch'ang-k'ing. Pauthier et Dabry de Thiersant ont traduit ce titre par „président du tribunal des rites", Legge par „directeur de la Cour des sacrifices”. Comme ce personnage est donné en syriaque comme archidiacre, le P. Heller (p. 56) suppose que $t^{\prime} a i$-tch'ang-k'ing signifie „président des saints usages” et équivaut à „archidiacre” pour lui, Pauthier, Dabry de Thiersant et Legge ont dû lire 党 t'ang, au lieu de 管 tch'ang, pour traduire comme ils l'ont fait. Il n'en est rien. La Cour des sacrifices est bien counue, et c'est une institution purement chinoise.

Addendum. - M. l'abbé Chabot, à qui j'ai communiqué les épreuves de cette note, me fait remarquer que mes hypothèses tendent à montrer que, dans la transcription syriaque de mots chinois, toutes les voyelles étaient notées, et que tel fut aussi l'usage dans

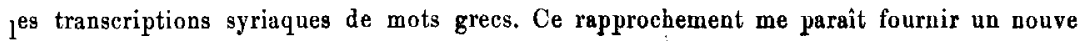

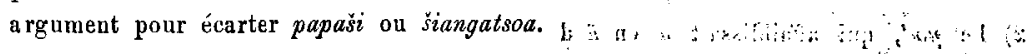

